\title{
Investigation of thickness and density dependence of thermal conductivity of Expanded Polystyrene insulation materials
}

\author{
Ákos Lakatos ${ }^{1, \text { a }}$, Ferenc Kalmár ${ }^{1, b}$ \\ University of Debrecen, Faculty of Engineering, \\ Department of Building Services and Building Engineering, Debrecen, Hungary \\ H-4028 Debrecen, Ótemető str. 2-4. \\ Tel:+36-52-415-155 \\ Fax:+36-52-415-155/77713 \\ aalakatos@eng.unideb.hu, bfkalmar@eng.unideb.hu \\ Keywords: thermal conductivity, hot plate method, Expanded Polystyrene
}

\begin{abstract}
Analysis of thermal conductivity of different insulation materials is very important in applications for passive and nearly zero houses. This paper presents the measurements results of the thermal conductivities of expanded polystyrene (EPS) insulation materials, with different thicknesses and air pore content. The measurements were achieved on pure (white) as well as graphite -enhanced (grey) EPS slabs, with a Holometrix type 2000 series heat flow meter after desiccating them in a Venticell (VC) 111 type drying apparatus to changeless weight. The densities of the white samples were varied from 10 to $26 \mathrm{~kg} / \mathrm{m}^{3}$ (EPS types 30-200 and grey), furthermore both the white ones and the grey $\left(14 \mathrm{~kg} / \mathrm{m}^{3}\right)$ samples with three different thicknesses $(5,8,10 \mathrm{~cm})$ were tested. In addition, experimental results for the density dependence of thermal conductivity at low densities are given. Thermal conductivity measurements of different white slabs in combination with grey slabs were executed also and the changes in the thermal conductivity values are presented.
\end{abstract}

\section{Introduction}

Expanded polystyrene is proved to be an excellent insulating medium which exhibits consistent thermal performance over the range of temperatures normally encountered in buildings. It is also used in a wide range of other areas including, packaging, buoyancy, panel cores, bean bags and civil engineering. Thermal conductivity is a key thermal transport property of materials. The importance of the measurements of the thermal conductivities of insulation materials are written by other researchers presented in [1-8]. Many types of insulation materials are available which differ with regard to thermal properties and many other material properties as well as cost. For calculating the R-values used to design building walls and roofs structures depends strongly on the thermal conductivity of insulation materials. Laboratory and prototype situations for measuring the thermal property values are strongly needed for the above mentioned calculations. [9]

\section{Material and methods}

The expanded polystyrene materials were produced in the Cellplast Plastic Ltd in Hajduszoboszló. The main steps through the sample preparations were the followings. After the preparation of the raw material, the samples were taken to the pre-expander and were heat-treated at $368 \mathrm{~K}$. From the pre-expander the dried materials were exposed to a half day conditioning. Then the moulding and the 
second conditioning of the semi-prepared samples came after. On completion of the sample preparing they can be dimensioned. Two different types of Expanded Polystyrene insulation materials were investigated. At first, the conventional pure (white) EPS, then the polystyrene enhanced with graphite, the so-called grey EPS, slabs were examined. The reasons and advantages of the graphite-doped samples are the effective usage during the construction of passive houses. Their application does not require any special procedures furthermore, one can reach lower heat losses of objects by using them, because their good thermal resistance properties.

The thermal conductivity measurements were carried out after drying the EPS samples in a VentiCell drying instrument to changeless weight [8]. With this device materials can be dried setting different air temperatures (up to $523 \mathrm{~K}$ ). It works with hot air circulation using an inbuilt ventilator [8]. For measuring the thermal conductivity of polystyrene samples a Lambda 2000 Heat flow meter (HFM) was applied. This equipment is designed to determine the thermal conductivity of insulation materials in accordance with standard ASTM C518 and ISO 8301 protocols. The white and the grey samples with $30 \mathrm{~cm} \times 30 \mathrm{~cm}$ area and with 5,8 and $10 \mathrm{~cm}$ height geometry were placed in the test section between two plates which are maintained at different temperatures $\left(\mathrm{T}_{1}=285 \mathrm{~K}\right.$ and $\mathrm{T}_{2}=295 \mathrm{~K}$, with $\mathrm{T}_{\text {mean }}=290 \mathrm{~K}$ ) during the test. Another measurement series were done with conflating the $5 \mathrm{~cm}$ thick white EPS slabs with a $5 \mathrm{~cm}$ thick grey sample.

Through the measurements in the Holometrix application, if the instrument is achieving thermal equilibrium and establishing a uniform temperature gradient throughout the sample, thermal conductivity is determined. To determine the thermal conductivity of a sample, three independent measurements were carried out. The thermal conductivity of analysed material was the average of the three measured values.

For understanding the measurement method of Holometrix Lambda equipment the following comments are indispensable. The magnitude of the heat flow (q) depends on several factors:

a) thermal conductivity of samples $(\lambda=\mathrm{k})$

b) the thickness of the specimen $(\Delta x)$

c) the temperature difference across the specimen $(\Delta \mathrm{T})$

d) the area through which the heat flows (A).

The Fourier heat flow equation (Eq. 1.) gives the relationship between these parameters when the test section reaches thermal equilibrium.

$$
q=\lambda A \frac{\Delta T}{\Delta x}
$$

One or two heat flow transducers measure the heat flow through the specimen. The signal of a heat flow transducer (in Volts (V)) is proportional to the heat flow through the transducer. In the Lambda 2000 HFM instrument, the area of the heat flow transducer represents the area through which the heat conduction is realized and it is the same for all specimens, therefore the heat flow will be (Eq. 2):

$$
q=N V
$$

where $\mathrm{N}$ is the calibration factor, relates to the voltage signal of the heat flow transducer to the heat flux through the specimen. For calibration of apparatus a 
fibrous glass board standard sample with $\lambda=0.054 \mathrm{~W} / \mathrm{mK}$ was used. Using Eq. 1 and Eq. 2 the heat conductivity can be determined (Eq. 3):

$$
\lambda=k=N \frac{V \Delta x}{\Delta T}
$$

From the measured thermal conductivity $(\lambda)$ coefficients one can calculate the thermal diffusion coefficient $\left(\mathrm{m}^{2} / \mathrm{s}\right)$, using (Eq. 4):

$$
D_{T}=\frac{\lambda}{\rho \bullet c}
$$

where, $\rho\left(\mathrm{kg} / \mathrm{m}^{3}\right)$ is the mass density, and $\mathrm{c}(\mathrm{J} / \mathrm{kg} * \mathrm{~K})$ is the specific heat (for polystyrene $\mathrm{c}=1460 \mathrm{~J} / \mathrm{kg}^{*} \mathrm{~K}$ ).

\section{Results and Discussion}

\section{a) The role of the density}

Thermal conductivities of the expanded polystyrene materials were investigated. The EPS slabs with different mass densities are collected in table 1 and presented on figure 1 . The decreasing thermal conductivity with decreasing density can be observed as published by others [1-7]. It is mainly caused by decreasing air content and decreasing size of the air inclusions, with increasing density. In this case the heat flow through the samples is directed by the conduction of the solid particles, furthermore the convection of air is getting insignificant.

Table 1.: The thermal conductivities and mass densities of pure white EPS slabs.

\begin{tabular}{|c|c|c|}
\hline $\begin{array}{c}\rho_{\mathrm{i}}, \text { Density } \\
\left(\mathrm{kg} / \mathrm{m}^{3}\right)\end{array}$ & $\lambda_{\mathrm{i}}$, Thermal conductivity $(\mathrm{W} / \mathrm{mK})$ & $\begin{array}{c}\mathrm{D}_{\mathrm{T}}, \text { Thermal Diffusion coefficient } \\
\left(\mathrm{m}^{2} / \mathrm{s}\right)\end{array}$ \\
\hline 10.221 & 0.04887 & $3.27 * 10^{-6}$ \\
\hline 12.124 & 0.04669 & $2.64 * 10^{-6}$ \\
\hline 14.45 & 0.04200 & $1.99^{*} 10^{-6}$ \\
\hline 15.57 & 0.04070 & $1.79^{*} 10^{-6}$ \\
\hline 16.73 & 0.03800 & $1.56^{*} 10^{-6}$ \\
\hline 17.638 & 0.03943 & $1.53^{*} 10^{-6}$ \\
\hline 21.445 & 0.03634 & $1.16^{*} 10^{-6}$ \\
\hline 22.514 & 0.03600 & $1.10^{*} 10^{-6}$ \\
\hline 22.548 & 0.03685 & $1.12^{*} 10^{-6}$ \\
\hline 23.581 & 0.03553 & $1.03^{*} 10^{-6}$ \\
\hline 25.446 & 0.03517 & $9.47 * 10^{-7}$ \\
\hline 25.964 & 0.03400 & $8.97 \mathrm{E}^{*} 10^{-7}$ \\
\hline
\end{tabular}

For estimating the interdependence between the mass density and the thermal conductivity two different linear fit were applied on both the low density, and the greater density values. The slope of either function shows unambiguous decreasing. For presenting the role of the density, so that the effect of air content a hypothetic calculation was carried out. If one fixes, both the density value of the 
sample with the greatest density $\left(\rho_{\max }=25.964 \mathrm{~kg} / \mathrm{m}^{3}\right)$ and its thermal conductivity value $\left(\lambda_{\min }=0,034 \mathrm{~W} / \mathrm{mK}\right)$ as comparative values and using the Eq. 5 and 6

$$
\begin{aligned}
& n(\%)=\frac{\rho_{\text {max }}-\rho_{i}}{\rho_{\text {max }}} \\
& \lambda(\%)=\frac{\lambda_{\text {min }}-\lambda_{i}}{\lambda_{\text {min }}}
\end{aligned}
$$

can estimate the "n: density factor" what gives us the result of the air content and pore size, furthermore one can reach the increase of thermal conductivity with decreasing density in percentage: $\lambda(\%)$. The values are collected in table 2 and plotted on figure 2 .

Table 2.: The density factors and the changes in the thermal conductivity

\begin{tabular}{|c|c|c|c|}
\hline $\mathrm{n}(\%)$ & $\lambda(\%)$ & $\mathrm{n}(\%)$ & $\lambda(\%)$ \\
\hline 60.634 & 43.733 & 32.067 & 15.961 \\
\hline 53.305 & 37.323 & 17.405 & 6.882 \\
\hline 44.346 & 23.529 & 13.288 & 5.882 \\
\hline 40.032 & 19.707 & 13.157 & 8.382 \\
\hline 35.565 & 11.765 & 9.178 & 4.510 \\
\hline 32.067 & 15.961 & 1.995 & 3.431 \\
\hline
\end{tabular}

From the values of table 2 one can see that there is increasing $\lambda \%$ with increasing density factor. Between the sample with $\rho_{\max }$ density and the sample with 10 $\mathrm{kg} / \mathrm{m}^{3}$ density about $60 \%$ difference can be observed. Belonging to this density difference value the thermal conductivity of the low density material is only $40 \%$ higher than $\lambda_{\min }$. However, for $40 \%$ density difference only $20 \%$ thermal conductivity change is measured.

\section{b) The role of the thickness of the samples}

As may be imagined, the thermal conductivity should not be a function of the sample thickness; after all we measured the thermal conductivity of the EPS samples with different thicknesses. The grey sample and, four another samples, from the pure EPS materials with different mass densities indicated in Table 3, were chosen and were tested with 8 and $10 \mathrm{~cm}$ thicknesses either. First of all, from table 3 and figure 3 the same phenomena can be seen, that for each thicknesses $(5,8$ and $10 \mathrm{~cm})$ the thermal conductivities are decreasing with increasing density, similarly as above mentioned. The lowest $\lambda$ values are belonging to the grey polystyrene material. It is the best EPS insulation material with the lowest density and the lowest thermal conductivity. From figure 3 in the slope of the lines a conspicuous break can be seen for all densities. The samples 
with $8 \mathrm{~cm}$ thickness show the highest thermal conductance. In general the $\lambda$ values belonging to the $10 \mathrm{~cm}$ thick samples are between the values of $\lambda_{5} \mathbf{~ c m}$ and $\lambda_{8} \mathbf{~ c m}$. If one calculates the differences between the $\lambda$ values of each EPS samples less than $8 \%$ deviation can be found, so that we don't attach too much physical importance to these phenomena. As may be known, the thermal conductivity does not depend on the thickness of the specimen. It can be mainly caused by the nature of the samples and the measurement method. An average value for all the samples with the same densities can be calculated.

Table 3.: The thermal conductivities regarding to different densities and different thicknesses.

\begin{tabular}{|c|c|c|c|c|}
\hline $\boldsymbol{\lambda}_{\mathbf{5} \mathbf{~ c m}}(\mathbf{W} / \mathbf{m K})$ & $\boldsymbol{\lambda}_{\mathbf{8} \mathbf{c m}}(\mathbf{W} / \mathbf{m K})$ & $\begin{array}{c}\boldsymbol{\lambda}_{\mathbf{1 0} \mathbf{c m}} \\
(\mathbf{W} / \mathbf{m K})\end{array}$ & $\begin{array}{c}\text { Density } \\
\left(\mathbf{k g} / \mathbf{m}^{3}\right)\end{array}$ & $\begin{array}{c}\text { Average } \\
(\mathbf{W} / \mathbf{m K})\end{array}$ \\
\hline 0.0420 & 0.0440 & 0.0430 & 12.2807 & 0.043 \\
\hline 0.0380 & 0.0407 & 0.0394 & 16.6472 & 0.0394 \\
\hline 0.0350 & 0.0369 & 0.036 & 22.8811 & 0.036 \\
\hline 0.0340 & 0.0352 & 0.0350 & 24.2848 & 0.0347 \\
\hline 0.0320 & 0.0336 & 0.0334 & 13.8042 & 0.033 \\
\hline
\end{tabular}

\section{c) The role of the grey samples}

In this section, we present the measured thermal conductivity values of each white samples in combination with a grey (graphite-enhanced) polystyrene sample. One of the best insulation properties of the grey EPS materials is its outstanding resistance against the heat radiation inside the sample, by its infrared reflector and absorber carbon particles. The insulating properties of the grey EPS slabs are 15 to $20 \%$ better than that of conventional white Expanded Polystyrene. If one measures together the $5 \mathrm{~cm}$ thick white EPS materials with a $5 \mathrm{~cm}$ grey EPS material, one can reach mixed thermal conductivity value of a $10 \mathrm{~cm}$ slab. Comparing the "lambda" values carried out in this way with the thermal conductivities presented in part 3.b), one can estimate an amelioration rate (in \%), indicated in Table 4. The change in the thermal conductivity in the highest degree is observed by the sample with 14 $\mathrm{kg} / \mathrm{m}^{3}$ mass density. Nearly marginal, (approximately 5\%), is the change in the thermal conductivity of the samples with greater than $20 \mathrm{~kg} / \mathrm{m}^{3}$ density. If one plots the changes in the thermal conductivity in function of the density of the original pure white samples an exponential function can be reached (see Figure 4.). On figure 4 one can see the distinct improvement in the "lambda" value in inverse ratio to the density.

Table 4.: The thermal conductivities of each mixed samples as well as the original values.

\begin{tabular}{|c|c|c|c|c|}
\hline & $\begin{array}{c}5 \mathrm{~cm}(14 \\
\left.\mathrm{kg} / \mathrm{m}^{3}\right)+5 \mathrm{~cm} \\
(\text { grey })\end{array}$ & $\begin{array}{c}5 \mathrm{~cm}(16.5 \\
\left.\mathrm{kg} / \mathrm{m}^{3}\right)+5 \mathrm{~cm} \\
(\mathrm{grey})\end{array}$ & $\begin{array}{c}5 \mathrm{~cm}(22 \\
\left.\mathrm{kg} / \mathrm{m}^{3}\right)+5 \mathrm{~cm} \\
(\text { grey })\end{array}$ & $\begin{array}{c}5 \mathrm{~cm}(25 \\
\left.\mathrm{kg} / \mathrm{m}^{3}\right)+5 \mathrm{~cm} \\
(\mathrm{grey})\end{array}$ \\
\hline $\begin{array}{c}\lambda \text { of } 10 \mathrm{~cm} \text { thick } \\
\text { samples }\end{array}$ & 0.0359 & 0.0352 & 0.0342 & 0.0335 \\
\hline
\end{tabular}




\begin{tabular}{|c|c|c|c|c|}
$\begin{array}{c}\text { Decrease in the } \lambda \\
(\%)\end{array}$ & 19.6 & 11.9 & 5.4 & 4.48 \\
\hline$\rho\left(\mathrm{kg} / \mathrm{m}^{3}\right)$ & 14 & 16.5 & 22 & 25 \\
\hline
\end{tabular}

\section{Conclusions}

The impact of mass density and sample thickness on thermal conductivity of EPS materials has been investigated by Holometrix equipment. Thermal conductivities of EPS various mass densities were measured at $290 \mathrm{~K}$ operating mean temperature. We found that thermal conductivity decreases with increasing density in this density range $\left(10 \mathrm{~kg} / \mathrm{m}^{3}-25 \mathrm{~kg} / \mathrm{m}^{3}\right)$ as before reported as us and others, from the "lambda" values thermal diffusion coefficients were calculated either.

A hypothetic model is created to estimate the density influenced changes in the thermal conductivity.

Thickness dependency examinations were done as well, although we did not observe any deviances in the thermal conductivity coefficient. Furthermore, we investigated the changes in the thermal conductivity of the white EPS materials in combination with grey polystyrene as well. An exponential function was given for the determination of the impact of the graphite-enhanced polystyrene material on the thermal conductivity of white EPS materials. However the thermal conductivity of the materials does not depend on the thicknesses of the specimens, but the $\mathrm{R}$ value strongly does. By calculating $\mathrm{R}$ values minimal thickness for the efficient thermal insulation should be about $10 \mathrm{~cm}$. Regarding to our results the optimal resistivity can be reached by the application of $5 \mathrm{~cm}$ white EPS 200 in combination with $5 \mathrm{~cm}$ EPS grey materials.

\section{Acknowledgements}

The authors gratefully acknowledge the support of Cellplast Plastic Ltd. (H-4200, Hajdúszoboszló, Szováti str $3 / \mathrm{b}$ ) for ensuring the polystyrene specimens. The work/publication is supported by the TÁMOP 4.2.1/B-09/1/KONV-2010-0007 project and TÁMOP 4.2.4. A/1-11-1-2012-0001 projects. The projects are co-financed by the European Union and the European Social Fund.

\section{References}

[1] Mar, J.D., Litovsky, E., Kleiman, J., (July 2008) Journal of Building Physics. vol. 32 no. $19-$ 31. Modeling and Database Development of Conductive and Apparent Thermal Conductivity of Moist Insulation Materials

[2] Yucel, K.T., Basyigit, C., Ozel, C. (2009) Thermal Insulation properties of expandedpolystyrene as construction and insulating materials Information on: http://zenonpanel.com.mk/al/wp- content/uploads/2009/06/Thermal-Insulation-properties.PDF. Date of download. 2011-11-28

[3] Hourston, D.J., Song, M., Hammiche, A., Pollock, H.M., Reading, M., Modulated differential scanning calorimetry: 2 . Studies of physical ageing in polystyrene. Polymer, 1996; 37, 2:243-247 [4] McCormick, H. W. Brower, F.M., Kin, L. The effect of molecular weight distribution on the physical properties of polystyrene. Journal of Polymer Science, 1959;39, 135:87-100.

[5] Xiao, M., Sun, L., Liu, J., Li, Y., Gong, K. Synthesis and properties of polystyrene/graphite nanocomposites. Polymer 2006; 43, 8: 2245-2248

[6] Morgan, A. B., Richard H. Harris, Takashi Kashiwagi, Leonard J. Chyall, Jeffrey W. Gilman Flammability of polystyrene layered silicate (clay) nanocomposites: Carbonaceous char formation. Fire and Materials 2002; 26, 6:247-253. 
[7] Mihlayanlar, E., Dilmac, S., Güner, A. Analysis of the effect of production process parameters and density of expanded polystyrene insulation boards on mechanical properties and thermal conductivity. Materials and design 2008; 29:344-352

[8] Á. Lakatos, F. Kalmár: Analysis of Water Sorption and Thermal Conductivity of Expanded Polystyrene Insulation Materials, Building Services Engineering Research and Technology, in print 2012.

[9] S.A. Al-Ajlan et al. Applied Thermal Engineering 26 (2006) 2184-2191 


\section{Figure Captions:}

Figure 1.: The thermal conductivity in function of mass density

Figure 2.: The percental change of the thermal conductivity in function of density factor

Figure 3.: The thickness dependence of the thermal conductivity

Figure 4.: The changes in the thermal conductivity in function of the mass density. 